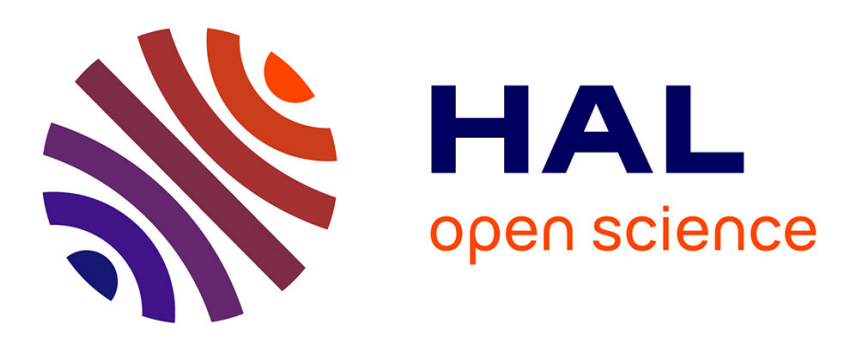

\title{
Impedance of a nanoantenna and a single quantum emitter
}

Jean-Jacques Greffet, Marine Laroche, François Marquier

\section{To cite this version:}

Jean-Jacques Greffet, Marine Laroche, François Marquier. Impedance of a nanoantenna and a single quantum emitter. Physical Review Letters, 2010, 105 (11), pp.117701. 10.1103/PhysRevLett.105.117701 . hal-00573254

\section{HAL Id: hal-00573254 \\ https://hal-iogs.archives-ouvertes.fr/hal-00573254}

Submitted on 4 Dec 2015

HAL is a multi-disciplinary open access archive for the deposit and dissemination of scientific research documents, whether they are published or not. The documents may come from teaching and research institutions in France or abroad, or from public or private research centers.
L'archive ouverte pluridisciplinaire HAL, est destinée au dépôt et à la diffusion de documents scientifiques de niveau recherche, publiés ou non, émanant des établissements d'enseignement et de recherche français ou étrangers, des laboratoires publics ou privés. 


\title{
Impedance of a Nanoantenna and a Single Quantum Emitter
}

\author{
Jean-Jacques Greffet, ${ }^{*}$ Marine Laroche, and François Marquier \\ Laboratoire Charles Fabry, Institut d'Optique, CNRS, Université Paris-Sud, RD128, F-91127 Palaiseau Cedex, France
}

(Received 21 April 2010; revised manuscript received 22 June 2010; published 9 September 2010)

\begin{abstract}
Antennas are widely used by electrical engineers to enhance the coupling between propagating waves and electric sources or detectors. It is thus tempting to develop an optical analog to tailor visible light emission or absorption by an atom or a molecule. This idea has been put forward recently and it has been demonstrated that both the radiative rate and the emission pattern of optical emitters can be modified by metallic nanostructures. In this Letter, we introduce the concept of impedance for a nanoantenna and for two-level systems or nanoparticles described by electric dipole moments. We show how these concepts can be used to reconcile different descriptions and also to optimize nanoantennas.
\end{abstract}

In general terms, an antenna is a device that allows us to control the coupling between propagating waves and a finite size detector or radiation source. Antennas working in the optics regime at nanometer scale have been demonstrated recently [1-8]. In radio waves, the so-called gain characterizes the directivity of the antenna and the resistance characterizes the emitted power $R_{R} I^{2} / 2$ where $I$ is the intensity in the antenna and $R_{R}$ is the radiation resistance. In optics, the far-field emission pattern has been calculated by many authors [9-12]. Understanding how a nanoantenna can increase the power emitted by an excited two-level system with a fixed energy $\hbar \omega$ is more subtle. A nanoantenna can change the emission rate. Thus, under continuous illumination, the energy emitted per second can be increased because the number of absorption or emission cycles increases. It has been known since Drexhage's pioneer work [13] that the emission rate of a two-level system is not an intrinsic property of a quantum system but depends on the environment. In the 1940s, Purcell [14] suggested to use a microcavity to modify the emission rate. This concept proved to be very useful and led to a large number of developments $[15,16]$ in quantum optics. The figure of merit used in this context is the so-called Purcell factor. More recently, the role of metallic nanostructures as nanoantennas has been demonstrated [1-7]. The analysis of these results is done in terms of modification of radiative decay rate $\Gamma_{R}$ and nonradiative decay rate $\Gamma_{N R}[9,10]$. Recently, Maier [17] has introduced an analysis of metallic nanostructures in terms of effective volume and quality factor to reconcile the microcavity point of view with the antenna point of view. This approach has been successfully employed to analyze the properties of a plasmonic resonator by Khurgin and colleagues [18]. As both emission by atoms and emission by antennas deal with emission of radiation by electrons, there must be a connection between the concepts of emission rate used for metallic nanoantennas, Purcell factor used for microcavities, and radiation resistance used for radio waves antennas. The purpose of this Letter is to introduce a general formalism that allows us to analyze microcavities and nanoantennas in the optical regime using the impedance concept. To this aim, a new definition of the impedance is needed. If the nanoantenna consists in two metallic rods with a gap [2], the voltage across the gap can be defined. It is also possible to define a current density using the so-called displacement current. The application of these concepts in nanooptics has been extensively discussed by Engheta's group who has introduced many novel ideas in a series of recent contributions [19-22]. However, this approach cannot be used when dealing with the coupling between a two-level system and a nanosphere or a microcavity in which gaps cannot be defined. Indeed, when the antenna is a single metallic nanosphere or a microcavity, no voltage difference $U$ and no current intensity $I$ can be defined so that the usual definition $U / I$ cannot be used.

We start by a heuristic introduction of the impedance of an optical antenna based on the analysis of the power emitted by an optical dipole. Let us consider the power $P_{0}$ transferred by the dipole to the optical field. Assuming an $\exp (-i \omega t)$ time dependence, the time averaged value is given by $P_{0}=\left\langle-\frac{d \mathbf{p}}{d t} \cdot \mathbf{E}\right\rangle=\frac{1}{2} \operatorname{Re}\left(i \omega \mathbf{p} \cdot \mathbf{E}^{*}\right)$. There is a clear similarity between the structure of this equation and the familiar form of the electrical power $P$ dissipated in a load $P=\frac{1}{2} \operatorname{Re}\left(I U^{*}\right)$. This suggests to introduce a linear relation between the dipole moment and the field that accounts for the power dissipation due to the antenna. Such a relation is given by the Green tensor that yields the field radiated at $\mathbf{r}_{0}$ by a dipole located at $\mathbf{r}_{0}$ :

$$
\mathbf{E}(\mathbf{r})=\stackrel{\leftrightarrow}{\mathbf{G}}\left(\mathbf{r}_{0}, \mathbf{r}_{0}, \omega\right) \cdot \mathbf{p}\left(\mathbf{r}_{0}\right)=\frac{\overleftrightarrow{\mathbf{G}}\left(\mathbf{r}_{0}, \mathbf{r}_{0}, \omega\right)}{-i \omega} \cdot\left[-i \omega \mathbf{p}\left(\mathbf{r}_{0}\right)\right]
$$

In the following, we assume that the dipole is oriented along the $z$ axis so that $\mathbf{p} \cdot \stackrel{\leftrightarrow}{\mathbf{G}}^{*}\left(\mathbf{r}_{0}, \mathbf{r}_{0}, \omega\right) \cdot \mathbf{p}^{*}=$ $|\mathbf{p}|^{2} G_{z z}^{*}\left(\mathbf{r}_{0}, \mathbf{r}_{0}, \omega\right)$. The energy transferred by the dipole to the field can be written in the form $P_{o}=$ $\frac{1}{2} \operatorname{Im}\left(\frac{G_{z z}\left(\mathbf{r}_{0}, \mathbf{r}_{0}, \omega\right)}{\omega}\right)\left|\omega \mathbf{p}\left(\mathbf{r}_{0}\right)\right|^{2}$. This has the structure of the power $\frac{1}{2} \operatorname{Re}\left(Z_{L}\right)|I|^{2}$ dissipated in a load $Z_{L}$. A comparison 
between the two forms of the power delivered by a source suggests the following identification:

$$
I \leftrightarrow-i \omega p_{z}\left(\mathbf{r}_{0}\right) \quad U \leftrightarrow-E_{z}\left(\mathbf{r}_{0}\right) \quad Z \leftrightarrow \frac{-i G_{z z}\left(\mathbf{r}_{0}, \mathbf{r}_{0}, \omega\right)}{\omega},
$$

where we have $U=Z I$. As for lumped elements, losses are given by the real part $R$ of the impedance $Z=R+i Y$. The resistive part of the impedance is $G_{z z}^{i} / \omega$ and accounts for both radiative losses and Joule losses. This result allows us to establish a connection with the quantum optics point of view. From Fermi's golden rule, we know that the spontaneous emission rate is proportional to the number of final states available and therefore to the local density of electromagnetic states. The latter is proportional to the imaginary part of the Green tensor [23-26] and therefore to the nanoantenna resistance. It is important to emphasize that this impedance has the dimension of $\Omega \cdot \mathrm{m}^{-2}$. It is thus an impedance per unit area. The difference stems from the fact that we use $-i \omega p_{z}$ (in $\mathrm{A} \cdot \mathrm{m}$ ) instead of $I$ (in $A$ ) and $-E_{z}$ (in $\mathrm{V} / \mathrm{m}$ ) instead of $U$ (in $\mathrm{V}$ ). We shall therefore use the term "specific impedance" hereafter. We note that this approach is similar to the so-called emf method introduced by Brillouin [27] in 1922 to derive the input impedance in the case of a wire antenna. For the sake of illustration, let us apply this tentative definition to simple and well-known systems: a vacuum, a microcavity, and a metallic nanosphere. The details of the calculation of the Green tensor for each case are given in the supplementary material [28].

We first consider emission by a dipole source in a vacuum. In the analogy, the radiating dipole is equivalent to a current source and the vacuum is equivalent to the load. According to the previous discussion, the impedance of the vacuum can be derived from the Green tensor. The imaginary part of the Green tensor is evaluated in Ref. [29] and is given by $G_{0 z z}^{i}\left(\mathbf{r}_{0}, \mathbf{r}_{0}, \omega\right)=\omega^{3} / 6 \pi \epsilon_{0} c^{3}$. Using this equation, we find: $P_{0}=\omega^{4}|p|^{2} / 12 \pi \epsilon_{0} c^{3}$. Hence, we have recovered the familiar power emitted by a dipole in a vacuum. It is important to note that the real part of the vacuum Green tensor $\overleftrightarrow{\mathbf{G}}_{0}\left(\mathbf{r}_{0}, \mathbf{r}, \omega\right)$ (or the imaginary part of the specific impedance) diverges when $\mathbf{r}$ reaches $\mathbf{r}_{0}$. This problem can be circumvented by including the real part of the Green tensor in the definition of the polarizability of the source as discussed in the supplementary material. In the following, the vacuum impedance will always be reduced to its resistive part $R_{0}$, the reactive part (real part of the vacuum Green tensor) being included in an effective polarizability of the source.

We can also apply the impedance definition to a microcavity. It is found that the impedance of the microcavity is equivalent to a parallel RLC circuit (see Fig. 1) with $R=$ $\frac{Q \omega}{\epsilon_{0} V_{\text {eff }} \omega_{r}^{2}}, L=\frac{1}{\epsilon_{0} V_{\text {eff }} \omega_{r}^{2}}, C=\epsilon_{0} V_{\text {eff }}$. The microcavity can thus be viewed as a notch filter with a large imaginary impedance at resonance. Let us note that the knowledge of $L$ and
$C$ can be used to derive the energy stored in the system. We stress that the Purcell factor of the microcavity is recovered in a natural way by taking the ratio of the vacuum and microcavity resistance. We now turn to the nanoantenna. For the sake of illustration, let us consider a spherical nanoparticle with radius $a$ and dielectric constant $\epsilon(\omega)$ given by Drude's model. The nanoantenna also appears to be an effective RLC parallel circuit. The effective mode volume of the nanoantenna $V_{\text {eff }}=\pi z^{6} / a^{3}$ can be much smaller than the typical value of a microcavity $(\lambda / 2 n)^{3}$. For instance, when using nanospheres with a radius equal to, e.g., $5 \mathrm{~nm}$ and a distance $z=10 \mathrm{~nm}$, we find $V_{\text {eff }}=$ $25 \times 10^{-6} \mu \mathrm{m}^{3}$. This result clearly illustrates the potential of nanoantennas in terms of Purcell factor. Yet, for practical application as an antenna, Purcell's factor is not the only parameter. A large part of the energy extracted from the emitter is converted into heat in the antenna. It is thus useful to introduce the radiative yield. The decay rate of the resonant mode can be split in two terms: $\gamma=\gamma_{R}+\gamma_{N R}$, where $\gamma_{R}$ and $\gamma_{N R}$ are the radiative and nonradiative decay rates, respectively. Since the decay rate is proportional to the inverse of the resistance, the equivalent circuit involves two parallel resistances. Finally, microcavities or nanoantennas at resonance can be modeled by a parallel RLC circuit, with two specific resistances, as shown in Fig. 1.

The radiative yield of the resonant mode can be written as a ratio of resistances: $\eta_{\mathrm{rm}}=\frac{\gamma_{R}}{\gamma_{R}+\gamma_{N R}}=\frac{R_{N R}}{R_{N R}+R_{R}}$. In conclusion, we have introduced a definition of the impedance that is based on the Green tensor. Hence, it is defined for any structure and involves only the knowledge of the field generated by a dipole at its own location. Although we have used simple models to illustrate this concept with analytical formulas, we point out that numerical simulations can be used to derive the Green tensor $G_{z z}\left(\mathbf{r}_{0}, \mathbf{r}_{0}, \omega\right)$ for more complex geometries.

In order to account for interactions between the emitter and the antenna, we again seek inspiration in the circuit formalism. We need to introduce the two-level system impedance in order to be able to account for coupling between the antenna and the two-level system. Defining an internal specific impedance for the source amounts to finding a linear relation between the induced dipole moment $\mathbf{p}^{\text {ind }}$ and the electric field. A quantum analysis of resonant scattering using Bloch equations [30,31] shows

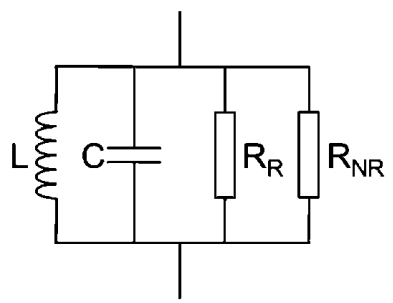

FIG. 1. Equivalent circuit RLC of a microcavity or a metallic nanoantenna. 
that for incident intensities much smaller than the saturation intensity, the interaction is essentially a coherent resonant scattering process that can be described using a polarizability: $\mathbf{p}^{\text {ind }}=\alpha \varepsilon_{0} \mathbf{E}$. We can thus write $\mathbf{E}=$ $Z_{\text {in }}\left(-i \omega \mathbf{p}^{\text {ind }}\right)$, where we have introduced the internal specific impedance of the source

$$
Z_{\text {in }}=\frac{i}{\omega \alpha \epsilon_{0}} .
$$

Let us consider for the sake of illustration the particular form of the polarizability for a two-level system given [24] by $\alpha(\omega)=\alpha_{0} /\left[\omega_{0}^{2}-\omega^{2}-i \gamma \omega\right]$ where $\alpha_{0}$ can be written using the oscillator strength $f$ as $\alpha_{0} \epsilon_{0}=\left(e^{2} / m\right) f$ and $\omega_{0}$ is the bare frequency of the dipole decoupled from all fields. We find $Z_{\text {in }}=\frac{\gamma}{\alpha_{0} \epsilon_{0}}+i \frac{\omega_{0}^{2}}{\alpha_{0} \epsilon_{0}} \frac{1}{\omega}-i \omega \frac{1}{\alpha_{0} \epsilon_{0}}$. Introducing the vacuum impedance $Z_{0}=\sqrt{\mu_{0} / \epsilon_{0}}$ and the extinction cross section at the resonant frequency $\sigma=$ $\frac{\omega_{0}}{c} \operatorname{Im}\left[\alpha\left(\omega_{0}\right)\right]$, we obtain:

$$
Z_{\text {in }}=\frac{Z_{0}}{\sigma}\left(1+i Q \frac{\omega_{0}}{\omega}-i Q \frac{\omega}{\omega_{0}}\right)
$$

where $Q$ is the quality factor of the resonance $Q=\frac{\omega_{0}}{\gamma}$. As shown in Fig. 2, this impedance has the structure of the impedance of a RLC series circuit.

We have introduced the specific impedance for a nanoantenna or a microcavity and a two-level system. We now derive the corresponding circuit equation. We start from the equation $p_{z}=\alpha \varepsilon_{0}\left[E_{\mathrm{ext}, z}+\left(i G_{0 z z}^{i}+S_{z z}\right) p_{z}\right]$, where $E_{\mathrm{ext}, z}$ is an external illuminating field and $S_{z z}=$ $G_{z z}-i G_{0 z z}^{i}$ accounts for the environment contribution to the Green tensor. This shows that the external source in the electrical analogy is a constant voltage source $U_{\text {ext }}=$ $-\left(Z_{\text {ant }}+R_{0}+Z_{\text {in }}\right) I$ [see Fig. 3(a)].

Note that the above equation is also valid for an atom or a small particle with polarizability $\alpha$ considered to be an absorber. We can use the equivalent circuit to derive the absorption in the load by using $R_{\text {in }}|I|^{2} / 2$. This allows us to maximize the absorption using the conjugate impedance matching condition. In order to discuss spontaneous emission, we cancel the external voltage source $\left(E_{\mathrm{ext}, z}=0\right)$, place the system in an initial state and study its time evolution [see Fig. 3(b)]. In the harmonic impedance description, the equation of the system is then $Z_{\text {ant }}(\omega)+$ $R_{0}+Z_{\text {in }}(\omega)=0$.

We consider now two applications: the emission of a quantum emitter in a microcavity, and the enhancement of the electroluminescence efficiency by silver nanoparticles.

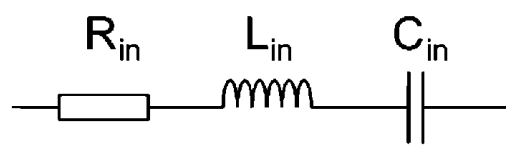

FIG. 2. Equivalent circuit of a quantum emitter. The lumped circuit elements are $R_{\mathrm{in}}=\frac{Z_{0}}{\sigma}, L_{\mathrm{in}}=\frac{Z}{\sigma} \frac{Q}{\omega_{0}}$ and $C_{\mathrm{in}}=\frac{\sigma}{Z_{0} \omega_{0} Q}$.
Regarding the quantum emitter in a microcavity, the previous equation has two solutions (strong coupling regime) or one solution (weak coupling regime). For an emitter without intrinsic losses $\left(\gamma=0\right.$, or $\left.R_{\text {in }} \rightarrow \infty\right)$, it is a simple matter to check that the eigenfrequency of the coupled system in the weak coupling regime is given by: $\omega \simeq \omega_{0}-$ $i \alpha_{0} \epsilon_{0} \omega_{0} Z / 2$, or $\omega \simeq \omega_{0}+\alpha_{0} \epsilon_{0} G_{z z}\left(\mathbf{r}_{0}, \mathbf{r}_{0}, \omega_{0}\right) / 2$. The real part of $G$ yields a frequency shift due to the interaction of the atom with the microcavity. The decay rate is the imaginary part of this complex frequency $\left.\alpha_{0} \epsilon_{0} G_{z z}^{i}\left(\mathbf{r}_{0}, \mathbf{r}_{0}, \omega_{0}\right)\right] / 2$. It shows that the two-level system lifetime is controlled by the local density of electromagnetic states as expected from Fermi's golden rule. Here we recover the standard result derived for a microcavity [15]. An advantage of the impedance formalism is that this result can be easily applied to any resonant structure.

We now consider the enhancement $F$ of the electroluminescence efficiency of a quantum well by silver nanoparticles already studied by Khurgin et al.. Without an antenna, the emitter decay rate through radiation in a vacuum is characterized by $R_{0}$ and the nonradiative processes are characterized by an internal resistance $R_{\text {in }}$. The radiative efficiency is then $\eta_{\text {rad }}=R_{0} /\left(R_{\text {in }}+R_{0}\right)$. We now study how this efficiency is improved by the presence of a nanoantenna. As seen above, the silver nanoparticle is modeled by a RLC parallel circuit with two resistances $R_{R}, R_{N R}$ in parallel. We introduce the nanoantenna radiative yield $\eta_{\mathrm{pr}}=\frac{R_{N R}}{R_{N R}+R_{R}}$ and the nanoantenna Purcell factor $F_{p}=\left(R_{0}+R\right) / R_{0}$. For $R \gg R_{0}$, we have $F_{p} \simeq R / R_{0}$. We now derive the radiative fluorescence efficiency with an antenna. If the emission frequency coincides with the nanoantenna resonance, this is simply given by the ratio of dissipated powers in specific resistances: $\frac{R_{0}+R \eta_{\mathrm{pr}}}{R_{0}+R+R_{\mathrm{in}}}$. It is then easy to recover the result given by Khurgin et al.

$$
F=\frac{1+F_{p} \eta_{\mathrm{pr}}}{1+F_{p} \eta_{\mathrm{rad}}}
$$

Note that the formalism allows us to account for the effect of the detuning of the antenna.

In summary, a definition of the impedance of a nanoantenna and of a single quantum emitter has been intro- (a)

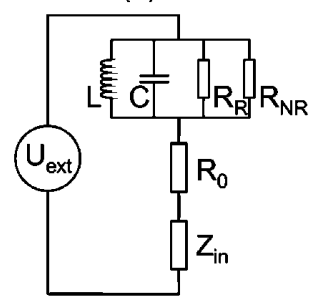

(b)

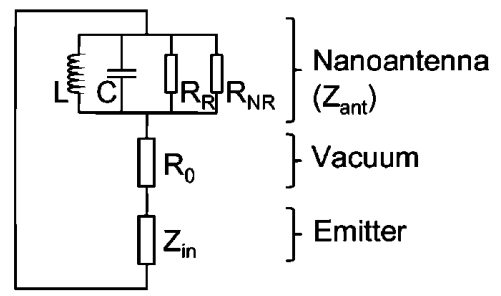

FIG. 3. Applications of the circuit analogy. (a) Absorption in the presence of an external field $\left(U_{\text {ext }}\right) . Z_{\text {in }}$ is the load impedance. (b) Spontaneous emission is modeled by looking for the complex eigenfrequency with $U_{\text {ext }}=0$. 
duced. These concepts allow us to establish a link between quantum optics and the electrical engineering points of view through the use of Green's tensor and the local density of states. Furthermore, this definition allows us to analyze in a unified framework the effect of the environment (nanoantenna, microcavity, waveguide, interfaces, etc.) on a quantum emitter. Explicit forms of the lumped elements of a microcavity and a simple two-level system have been derived. In summary, the concept of nanoantenna impedance provides both a deeper understanding of nanoantennas and a practical tool for the design and quantitative characterization of nanoantennas.

The authors acknowledge the support of Agence Nationale de la Recherche through the LAPSUS and Carnot Plasmonique projects. Jean-Jacques Greffet is grateful to Dieter Pohl for providing the inspiration for this work. The authors thank Rémi Carminati for stimulating discussions.

*jean-jacques.greffet@institutoptique.fr

†http://www.naphel.u-psud.fr/

[1] J. N. Farahni, D. W. Pohl, H. J. Eisler, and B. Hecht, Phys. Rev. Lett. 95, 017402 (2005).

[2] P. Mühlschlegel, H.-J. Eisler, O. J. F. Martin, D. W. Pohl, and B. Hecht, Science 308, 1607 (2005).

[3] K. B. Crozier, A. Sundaramurthy, G. S. Kino, and C. F. Quate, J. Appl. Phys. 94, 4632 (2003).

[4] P. J. Schuck, D. P. Fromm, A. Sundaramurthy, G. S. Kino, and W. E. Moerner, Phys. Rev. Lett. 94, 017402 (2005).

[5] P. Anger, P. Bharadwaj, and L. Novotny, Phys. Rev. Lett. 96, 113002 (2006).

[6] S. Kühn, U. Hakanson, L. Rigobete, and V. Sandoghdar, Phys. Rev. Lett. 97, 017402 (2006).

[7] J. J. Greffet, Science 308, 1561 (2005).

[8] P. Bharadwaj, B. Deutsch, and L. Novotny, Adv. Opt. Photon. 1, 438 (2009).

[9] L. Novotny, Appl. Phys. Lett. 69, 3806 (1996).

[10] M. Thomas, R. Carminati, J.-J. Greffet, and J. R. AriasGonzalez, Appl. Phys. Lett. 85, 3863 (2004).
[11] T. H. Taminiau, R. J. Moerland, and F. B. Segerink et al., Nano Lett. 7, 28 (2007).

[12] J. Li, A. Salandrino, and N. Engheta, Phys. Rev. B 79, 195104 (2009).

[13] K. H. Drexhage in Progress in Optics, edited by E. Wolf (North Holland, Amsterdam, 1974), p. 163.

[14] E. M. Purcell, Phys. Rev. 69, 681 (1946).

[15] S. Haroche, in Fundamental Systems in Quantum Optics, Proceedings of the Les Houches Summer School, Session LIII, edited by, J. Dalibard et al. (Elsevier Science Publishers, Amsterdam, 1992).

[16] H. Benisty, J. M. Gérard, R. Houdré, J. Rarity, and C. Weisbuch, Lectures Summer School Cargèse (Springer, Berlin, 1998).

[17] S. A. Maier, Opt. Express 14, 1957 (2006).

[18] J. B. Khurgin, G. Sun, and R. A. Soref, Appl. Phys. Lett. 93, 021120 (2008).

[19] N. Engheta, Science 317, 1698 (2007).

[20] A. Alu and N. Engheta, Nat. Photon. 2, 307 (2008).

[21] A. Alu and N. Engheta, Phys. Rev. Lett. 101, 043901 (2008).

[22] A. Alu and N. Engheta, Phys. Rev. B 78, 195111 (2008).

[23] R. Sprik, B. A. van Tiggelen, and A. Lagendijk, Europhys. Lett. 35, 265 (1996).

[24] L. Novotny and B. Hecht, Principles of Nano-Optics (Cambridge University Press, Cambridge, 2006).

[25] K. Joulain, R. Carminati, J.-P. Mulet, and J.-J. Greffet, Phys. Rev. B 68, 245405 (2003).

[26] Y. De Wilde, F. Formanek, R. Carminati, B. Gralak, P.-A. Lemoine, K. Joulain, J. P. Mulet, Y. Chen, and J. J. Greffet, Nature (London) 444, 740 (2006).

[27] L. Brillouin, Radioelectricité 3, 147 (1922).

[28] See supplementary material at http://link.aps.org/ supplemental/10.1103/PhysRevLett.105.117701 for a detailed analysis of the Green tensor of a microcavity and a nanoantenna as well as a discussion of the link between singularity and polarizability.

[29] R. Carminati, J. J. Greffet, C. Henkel, and J. M. Vigoureux, Opt. Commun. 261, 368 (2006).

[30] R. Loudon, The Quantum Theory of Light (Oxford University Press, Oxford, 2000).

[31] B. R. Mollow, Phys. Rev. 188, 1969 (1969). 